\title{
Hı properties and star formation history of a fly-by pair of blue compact dwarf galaxies *
}

\author{
Jinhyub Kim ${ }^{1}$, Aeree Chung ${ }^{1,2,3}$, O. Ivy Wong ${ }^{4}$, Bumhyun Lee ${ }^{1}$, Eon-Chang Sung ${ }^{5}$, and Lister Staveley-Smith ${ }^{4,6}$ \\ 1 Department of Astronomy, Yonsei University, 50 Yonsei-ro, Seodaemun-gu, 03722 Seoul, Korea \\ e-mail: achung@yonsei.ac.kr \\ 2 Yonsei University Observatory, Yonsei University, 50 Yonsei-ro, Seodaemun-gu, 03722 Seoul, Korea \\ 3 Joint ALMA Observatory, Alonso de Córdova 3107, Vitacura, Santiago, Chile \\ ${ }^{4}$ International Centre for Radio Astronomy Research (ICRAR), University of Western Australia, 35 Stirling Highway, Crawley, \\ Western Australia 6009, Australia \\ 5 Korea Astronomy and Space Science Institute 776, Daedeokdae-ro, Yuseong-gu, 34055 Daejeon, Korea \\ ${ }^{6}$ ARC Centre of Excellence for All-sky Astrophysics (CAASTRO), Australia
}

Received 20 February 2017 / Accepted 9 May 2017

\begin{abstract}
A fly-by interaction has been suggested to be one of the major explanations for enhanced star formation in blue compact dwarf (BCD) galaxies, yet no direct evidence for this scenario has been found to date. In the HI Parkes all-sky survey (HIPASS), ESO 435-IG 020 and ESO 435-G 016, a BCD pair were found in a common, extended gas envelope of atomic hydrogen, providing an ideal case to test the hypothesis that the starburst in BCDs can be indeed triggered by a fly-by interaction. Using high-resolution data from the Australia Telescope Compact Array (ATCA), we investigated HI properties and the spectral energy distribution (SED) of the BCD pair to study their interaction and star formation histories. The high-resolution HI data of both BCDs reveal a number of peculiarities, which are suggestive of tidal perturbation. Meanwhile, $40 \%$ of the HIPASS flux is not accounted for in the ATCA observations with no $\mathrm{HI}$ gas bridge found between the two BCDs. Intriguingly, in the residual of the HIPASS and the ATCA data, $\sim 10 \%$ of the missing flux appears to be located between the two BCDs. While the SED-based age of the most dominant young stellar population is old enough to have originated from the interaction with any neighbors (including the other of the two BCDs), the most recent star formation activity traced by strong $\mathrm{H} \alpha$ emission in ESO 435-IG 020 and the shear motion of gas in ESO 435-G 016, suggest a more recent or current tidal interaction. Based on these and the residual emission between the HIPASS and the ATCA data, we propose an interaction between the two BCDs as the origin of their recently enhanced star formation activity. The shear motion on the gas disk, potentially with re-accretion of the stripped gas, could be responsible for the active star formation in this BCD pair.
\end{abstract}

Key words. galaxies: individual: ESO 435-020 - galaxies: individual: ESO 435-016 - galaxies: interactions - galaxies: ISM galaxies: kinematics and dynamics - galaxies: starburst

\section{Introduction}

Blue compact dwarf galaxies (BCDs) are low-mass systems characterized by active star formation in compact regions and strong narrow emission lines superposed on a flat stellar continuum (e.g., Salzer et al. 1989; Kehrig et al. 2004). They were originally considered as either nascent or immature galaxies that had recently undergone intense bursts of star formation (Searle et al. 1973). However, it turns out that they also contain stellar populations older than $\sim 1$ Gyr as shown by a number of optical and near-IR studies (Thuan 1983; Papaderos et al. 1996; Aloisi et al. 1999), implying that BCDs are little galaxy fossils where starbursts have been recently triggered.

The low metallicity of BCDs $\left(1 / 50 \lesssim Z / Z_{\odot} \lesssim 1 / 10\right.$; Kunth \& Östlin 2000) also suggests that they have not always maintained their current star formation rate $\left(\sim 0.1 M_{\odot} \mathrm{yr}^{-1}\right.$; Hopkins et al. 2002). Indeed, the neutral hydrogen gas mass found in very gasrich BCDs is typically $10^{8} M_{\odot}$ (Thuan \& Martin 1981), which will require 1 Gyr to be consumed entirely if they keep up with

$\star$ The reduced datacube (FITS file) is only available at the CDS via anonymous ftp to cdsarc.u-strasbg. fr (130.79.128.5) or via http://cdsarc.u-strasbg.fr/viz-bin/qcat?]/A+A/605/A54 the current star formation rate (Thuan 1983). Instead, a number of models have shown that dwarf galaxies may go through a "BCD phase" due to the gas infall (e.g., Verbeke et al. 2014), merging with another dwarf galaxy (e.g., Di Matteo et al. 2007; Bekki 2008; Cloet-Osselaer et al. 2014), or fly-by interaction (e.g., Icke 1985; Pustilnik et al. 2001).

Those scenarios have been also tested observationally in a wide range of wavelengths from optical through to radio. For example, merging signatures or the presence of companions have been confirmed in $\mathrm{H} \alpha$ (e.g., Östlin et al. 1999; Mendez \& Esteban 2000; López-Sánchez \& Esteban 2009) and deep optical imaging studies (e.g., Rich et al. 2012). In addition, HI imaging has been particularly useful in finding the evidence for tidal interactions in BCDs (e.g., van Zee et al. 1998; Chengalur et al. 2006; Ekta et al. 2006; Lelli et al. 2012; López-Sánchez et al. 2012; Nidever et al. 2013). Approximately $57 \%$ of BCDs have been found with gas-rich but optically faint dwarf nearby companions (Taylor 1997), and the fraction increases up to $80 \%$ when BCDs with signatures of tidal interaction including merging are included (Pustilnik et al. 2001). All these observations support the hypothesis that the bursts of star formation in BCDs can be induced externally. 
On the other hand, there are cases of BCD pairs with separation larger than a few tens kpc such as UM 461/462 $(d \sim 68 \mathrm{kpc}$; Taylor et al. 1995), ESO 338-004/004B $(d \sim$ 72 kpc; Cannon et al. 2004), ESO 400-043/043B ( $d \sim 70 \mathrm{kpc}$; Östlin et al. 1999, 2001), and IRAS 08339+6517/2MASX J08380769+6508579 ( $d \sim 56 \mathrm{kpc}$; Cannon et al. 2004). For those BCD pairs, it is more difficult to prove that the two galaxies are or have been tidally interacting with each other. And even if this is so, how their "fly-by" interaction could lead to the recent active star formation is still questionable. Considering that there is quite a high fraction of BCD galaxies that are not in the process of merging ( $60 \%$, Pustilnik et al. 2001; Sung et al. 2002), it is important to show that they can indeed originate from fly-by interactions.

A pair of BCDs, ESO 435-IG 020 and ESO 435-G 016, separated by $\sim 80 \mathrm{kpc}$ in projection at similar distances ( $\sim 9 \mathrm{Mpc}$; Kennicutt et al. 2008), is one such example. Based on the fact that both galaxies appear to be highly disturbed (Mendez \& Esteban 2000; Rothberg \& Joseph 2004) in the optical observations, Sung et al. (2002) have classified this pair as "detached interacting BCDs", proposing that their peculiar optical morphology originates from tidal interaction with each other. Intriguingly, a large neutral hydrogen gas envelope has been revealed in the Hi Parkes all sky survey (HIPASS; Meyer et al. 2004), which is found to be covering both galaxies (see Fig. 1). Their optical morphologies and the common HI envelope make this pair a good candidate for the case of a BCD pair with fly-by interaction origin.

Considering the distance between the two galaxies, however, it is difficult to conclude whether HI gas associated with individual galaxies simply appears to be connected due to the large HIPASS beam, which is comparable to the separation of the pair on the sky, or whether they are indeed an interacting pair. In order to test the hypothesis that 1) these two dwarfs are going (or have gone) through a fly-by interaction; and 2) their interaction is the cause of their recent star formation activity, we followed up this region in HI with a higher resolution using the Australia Telescope Compact Array (ATCA). In this work, we probe detailed HI morphology and kinematics of the ESO 435-IG 020/016 pair and their star formation properties to investigate the role of a fly-by interaction as one of the important origins for quite a large fraction of the BCD population including our targets.

This paper is organized as follows. In Sect. 2, general properties of ESO 435-G 016/020 pair are introduced. In Sect. 3, the ATCA HI observation and data reduction are described. In Sect. 4, the HI morphology and kinematics of individual galaxies are presented. In Sect. 5, the presence of intergalactic HI between the pair is inspected. Also, the star formation and interaction histories of the pair are discussed. In Sect. 6, we summarize and conclude.

\section{ESO 435-IG 020 and ESO 435-G 016}

Both galaxies are small (a few kpc in diameter) and faint $\left(M_{B} \lesssim\right.$ $-18.5 \mathrm{mag}$ ) with strong, sharp, and narrow emission lines on a blue continuum, and both are classified as blue compact dwarf (BCD) galaxies (Sung et al. 2002). They are found at a similar redshift $\left(\sim 970-980 \mathrm{~km} \mathrm{~s}^{-1}\right)$ with an angular separation of $\sim 30$ arcmin, which corresponds to $\sim 80 \mathrm{kpc}$, adopting a $9 \mathrm{Mpc}$ distance to the pair (Kennicutt et al. 2008).

ESO 435-IG 020 has a compact core surrounded by an asymmetric, elongated stellar envelope. It was classified as an "interacting" system in the ESO atlas, as hinted by the

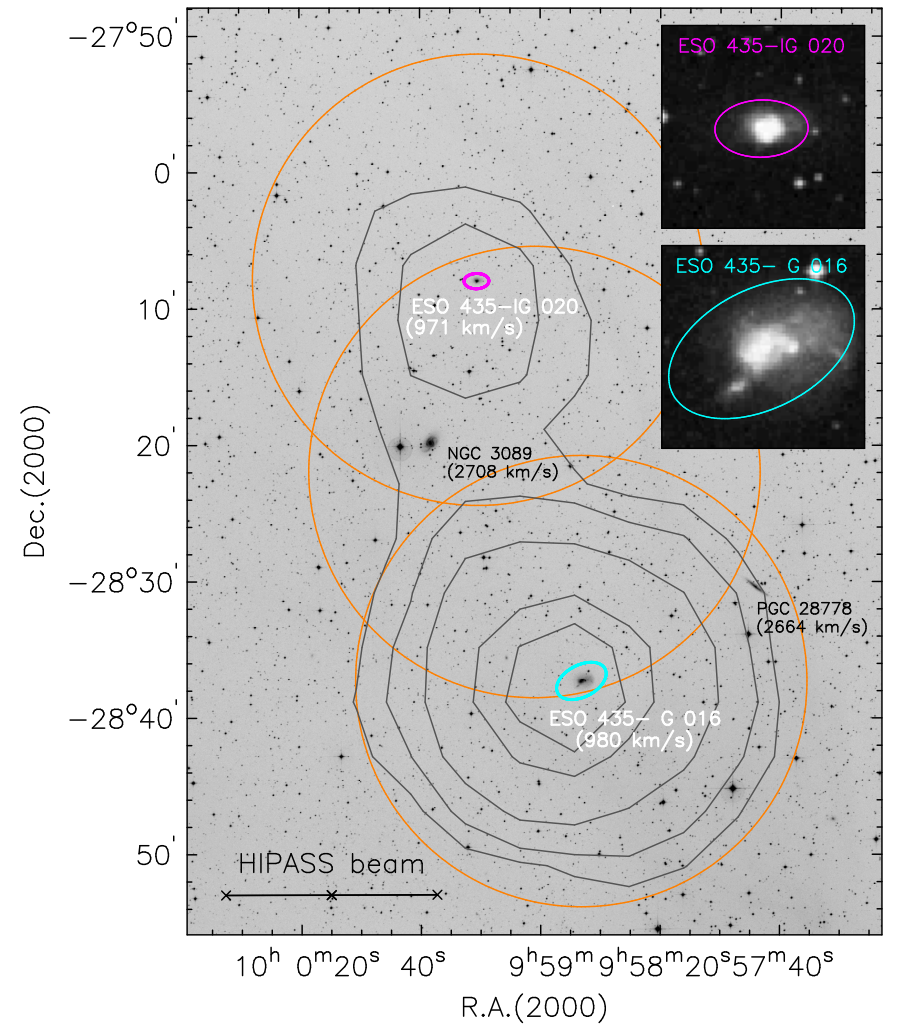

Fig. 1. HIPASS HI column density contours are overlaid on the DSS2 (Digital Sky Survey 2) red optical image. Two small colored ellipses represent the optical size, position angle (PA), and the inclination of our targets, taken from de Vaucouleurs et al. (1991, RC3). ESO cata$\log$ names of our BCDs are presented in white with their systemic velocity. Two other optically identified galaxies in this field, which are background systems, are also annotated in black. The HIPASS HI contour levels are $(3,6,12,24,30) \times 10^{18} \mathrm{~cm}^{-2}$. The primary beam of the Parkes telescope $\left(14^{\prime}\right)$ is shown at the lower left corner. Three ATCA pointings, each of which corresponds to the size of the ATCA primary beam $(\approx 33.1$ arcmin in diameter) are indicated by the orange line. On the top right corner, the zoom-in DSS2 blue images of individual galaxies are presented. Each panel is the size of $2 \times 2 \operatorname{arcmin}^{2}$. Again, the colored ellipse represents the optical size and PA of each galaxy (de Vaucouleurs et al. 1991).

catalog nomenclature (i.e., IG = interacting galaxy or galaxies Holmberg et al. 1974). Doublier et al. (1999) also found its brightness distribution to follow $r^{1 / 4}$ rather than an exponential profile, which is suggestive of (partial) violent relaxation after merging.

ESO 435-G 016 also has a diffuse stellar envelope which is off-center from the bright component. This galaxy has two sharp stellar tails; one inside the diffuse component in the west and a more distinctive tail pointing southeast. Based on these features, Rothberg \& Joseph (2004) identified this system as a merger remnant (it is referred to as AM 0956-282 in their work), although it was not classified as "interacting" in the ESO cata$\log$ (Holmberg et al. 1974). However, this galaxy is distinct from the rest of their sample in size and luminosity. It is the smallest among the sample of Rothberg \& Joseph (2004), being less luminous by 1.75 mag compared to the next faint galaxy in their sample, and fainter by 3 mag or more than the rest of the sample. Therefore, even if ESO 435-G 016 is a merger remnant, it is likely to be the result of merging between tiny galaxies like ESO 435-IG 020, which is even smaller. 
Table 1. Optical properties of the pair.

\begin{tabular}{lccccccccc}
\hline \hline Galaxy & $\begin{array}{c}\text { RA } \\
(\mathrm{J} 2000)\end{array}$ & $\begin{array}{c}\text { Dec } \\
(\mathrm{J} 2000)\end{array}$ & $\begin{array}{c}\text { Distance } \\
\text { Mpc }\end{array}$ & $\begin{array}{c}c z \\
\mathrm{~km} \mathrm{~s}^{-1}\end{array}$ & $\begin{array}{c}M_{B} \\
\mathrm{mag}\end{array}$ & $\begin{array}{c}M_{*} \\
10^{9} M_{\odot}\end{array}$ & $\begin{array}{c}D_{25} \\
\operatorname{arcsec}\end{array}$ & $\begin{array}{c}r_{25} \\
(b / a)_{25}\end{array}$ & $\begin{array}{c}\text { PA } \\
\mathrm{deg}\end{array}$ \\
\hline ESO 435-IG 020 & $09^{\mathrm{h}} 59^{\mathrm{m}} 21.2^{\mathrm{s}}$ & $-28^{\circ} 08^{\prime} 00^{\prime \prime}$ & 9.0 & 971 & -15.67 & 0.26 & 49.90 & 0.71 & 91.7 \\
ESO 435-G 016 & $09^{\mathrm{h}} 58^{\mathrm{m}} 46.2^{\mathrm{s}}$ & $-28^{\circ} 37^{\prime} 19^{\prime \prime}$ & 9.1 & 980 & -16.46 & 2.34 & 99.60 & 0.68 & 117 \\
\hline
\end{tabular}

Notes. Information on the galaxy coordinates (RA, Dec) and radial velocities comes from de Vaucouleurs et al. (1991, RC3). B-band luminosities, stellar masses, optical extents, and distances are taken from Kennicutt et al. (2008), and Fisher \& Drory (2011). Distances are corrected for the relative motion to the centroid of the Local Group (Kennicutt et al. 2008).

Alternatively, as Sung et al. (2002) suggested, the peculiarities of ESO 435-IG 020 and ESO 435-G 016 could be the result of tidal perturbation with each other. Sung et al. (2002) also proposed that fly-by interaction between the two galaxies could be the origin of their recent bursts of star formation; we aim to investigate this possibility in this work. General properties of the pair can be found in Table 1.

\section{ATCA observation and data reduction}

The HI observations were conducted on March 27 and 282002 with the ATCA in EW367 array configuration (project ID of C1024). The ATCA primary beam is $33.1 \mathrm{arcmin}$ at $1416 \mathrm{MHz}$, which is comparable to the angular distance between the pair of galaxies of $\sim 30.3$ arcmin. With a single pointing, the sensitivity at the locations of the galaxies hence drops by a factor of $\sim 1.8$, and therefore we had two extra pointings centered on each galaxy in order to keep the desired sensitivity toward the pair. The observing frequency was centered on $1416 \mathrm{MHz}$ with a total bandwidth of $8 \mathrm{MHz}$, which is configured into 512 channels, yielding a channel width of $3.3 \mathrm{~km} \mathrm{~s}^{-1}$.

We calibrated the data with a software package, Miriad (Sault et al. 1995). For flux and bandpass calibration, PKS 1934638 (14.87 Jy at the observing frequency) was used. As a phase calibrator, we observed PKS 1015-314 every 45 min during our observations. Before making an HI cube, the continuum was subtracted by applying a linear fit to the $u v$ data for a range of line free channels at both ends of the band. A final HI cube was generated using an AIPS $^{1}$ command IMAGR. In order to maximize the sensitivity while keeping a reasonable synthesized beam size and shape, we set robust $=0$ in IMAGR, the middle of the natural and uniform weighting scheme (Briggs 1995). Three points were stitched together by FLATN and corrected for the sensitivity loss corresponding to the primary beam shape. The final cube was smoothed to $5 \mathrm{~km} \mathrm{~s}^{-1}$ of velocity resolution to achieve a higher signal-to-noise ratio.

The total intensity HI map and the intensity weighted velocity field were produced using the task MOMNT in AIPS. It allows us to create a smoothed mask which helps to maximize the signal-to-noise ratio in the integrated map. For a mask, we adopted Gaussian and Hanning smoothing schemes in the spatial and velocity domains, respectively.

\section{Results}

\subsection{Integrated HI flux}

In the ATCA data, the pair is resolved into separate structures with the total HI flux of $(15.9 \pm 0.1) \mathrm{Jy} \mathrm{km} \mathrm{s}^{-1}$ and

\footnotetext{
1 Astronomical Image Processing System, http://www . aips . nrao. edu
}

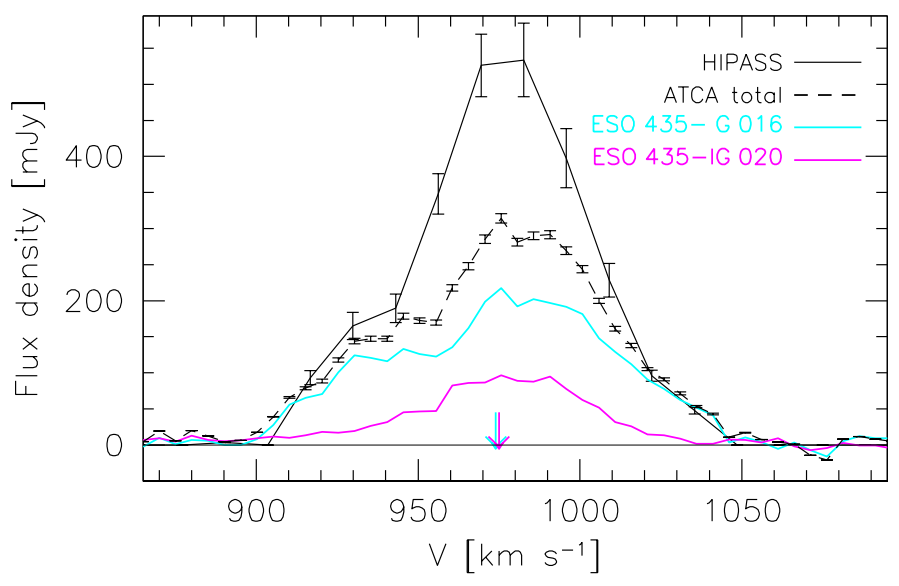

Fig. 2. Comparison of HI spectra. Flux densities of individual galaxies are shown in color and the integrated density of the two galaxies is indicated by the dashed black line. A black solid line represents the HIPASS flux density of the pair. The systemic velocity of each galaxy determined within a stellar disk is shown by a downward arrow of the same color as the HI spectrum of galaxies.

$(5.7 \pm 0.2) \mathrm{Jy} \mathrm{km} \mathrm{s}^{-1}$ associated with ESO 435-G 016 and ESO 435-IG 020, respectively. For ESO 435-G 016, the ATCA flux is only $58 \%$ of the previous single-dish measurement $\left(27.6 \pm 3.1 \mathrm{Jy} \mathrm{km} \mathrm{s}^{-1}\right.$, Parkes telescope; Koribalski et al. 2004), while for ESO 435-IG 020, we recover all the single-dish flux $\left(5.4 \pm 0.5 \mathrm{Jy} \mathrm{km} \mathrm{s}^{-1}\right.$, Nançay telescope; Theureau et al. 2005). The HI profiles from our ATCA observations are compared with the HIPASS HI spectrum in Fig. 2. The total ATCA flux of the pair is $\sim 61 \%$ of the HIPASS flux $\left(\approx 35 \mathrm{Jy} \mathrm{km} \mathrm{s}^{-1}\right)$.

Indeed, the HI column density sensitivity of our ATCA observation is not quite comparable to the HIPASS sensitivity (HIPASS versus ATCA $\approx 3 \times 10^{18} \mathrm{~cm}^{-2}$ vs. $1 \times 10^{19} \mathrm{~cm}^{-2}$ in $3 \sigma$ for $\Delta=50 \mathrm{~km} \mathrm{~s}^{-1}$ ), and hence most faint emission is likely to be missed from the ATCA data. In addition, the largest angular structure detectable using the ATCA EW367 at this wavelength is $\sim 16$ arcmin or so, while the HI envelope covering the pair found in the HIPASS image is much larger in extent ( $\sim 50$ arcmin), and the very extended features could have been resolved out. The presence of intergalactic gas is tested and further discussed in Sect. 5.

\subsection{High resolution $\mathrm{HI}$ morphology and kinematics}

The ATCA HI channel maps and the intensity maps are shown in Figs. 3 and 4. In the high resolution ATCA imaging data, we do not find any continuous HI gas emission between the pair. However, resolved structures reveal detailed features associated with individual galaxies. 


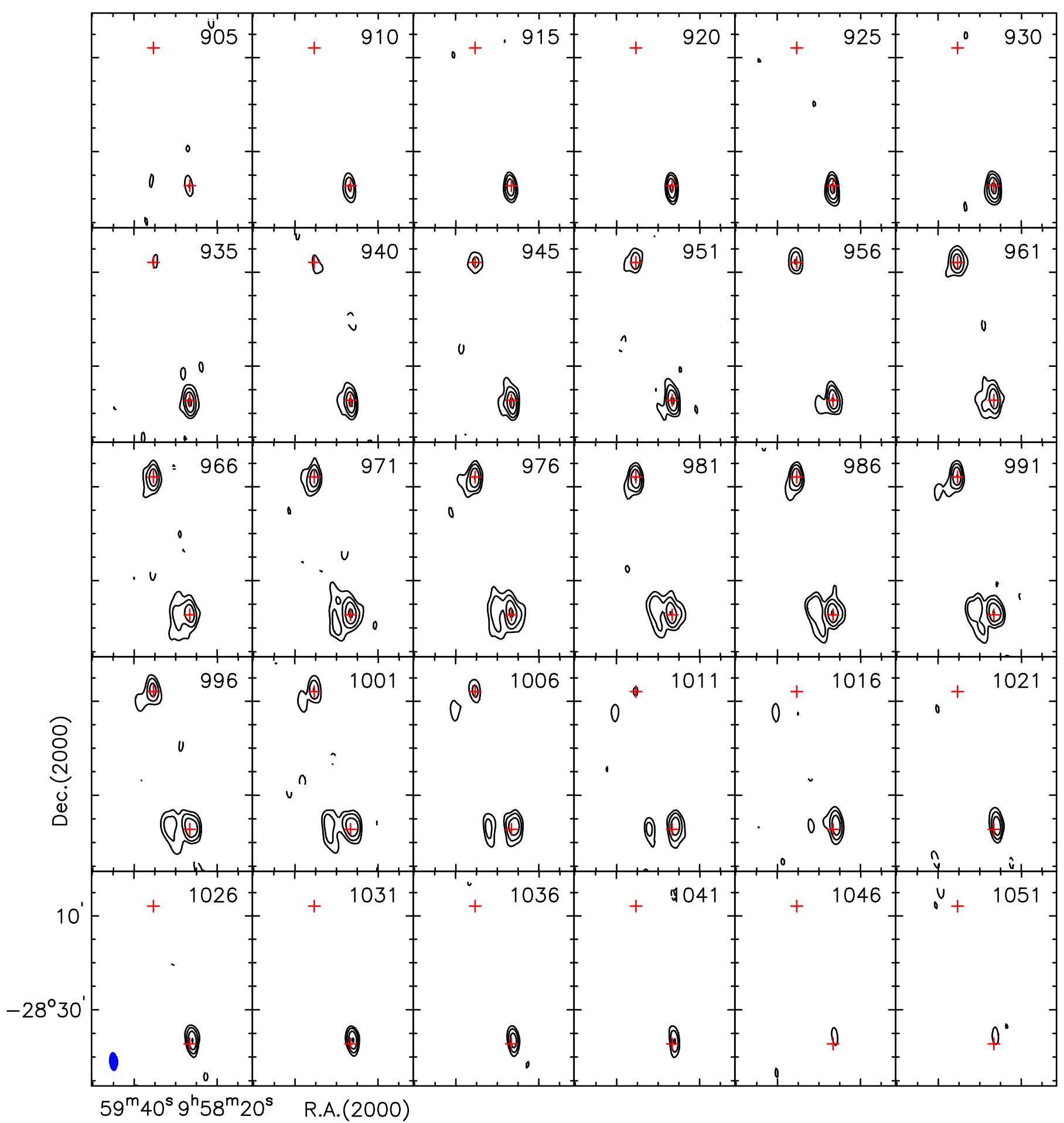

Fig. 3. ATCA HI channel maps. HI contour levels are $(-6,-3,3,6,12,24, \ldots) \times \sigma$ (negative values in dashed line), where $\sigma=1.2 \mathrm{mJy}^{\mathrm{m}}$ beam ${ }^{-1}$ per $5 \mathrm{~km} \mathrm{~s}^{-1}$ channel. Each panel is centered on the middle of two galaxies with the size of $\sim 60 \times 120 \mathrm{kpc}^{2}$ assuming a distance of 9 Mpc. Red crosses represent the optical center of individual galaxies. The velocity of the channel is shown on the top right of each panel in $\mathrm{km} \mathrm{s}^{-1}$. The ATCA synthesized beam $(218 \operatorname{arcsec} \times 85$ arcsec $)$ is indicated by a blue solid ellipse on the bottom left channel with its coordinates.

Both galaxies are found with a very extended gas disk. The largest feature is more extended than $3 \times D_{25}$, where $D_{25}$ is the optical size measured at 25 -mag $\operatorname{arcsec}^{-2}$ in $B$-band (de Vaucouleurs et al. 1991, RC3). This relative extent is comparable to those of BCDs located in low-density environments (e.g., van Zee et al. 1998). The HI gas is quite symmetric out to a few times of $D_{25}$, with the peak coinciding with the optical center within $<1 \mathrm{kpc}$. In the outskirts of the gas disk however, the gas morphology becomes highly irregular and asymmetric. ESO 435-IG 020 shows a long gas tail of $\sim 11 \mathrm{kpc}$, pointing to the southeast. The HI flux along the tail is measured to be $\sim 20 \%$ of the total flux of this system. In the case of ESO 435-G 016, a very extended structure is found mostly on the east side of the galaxy, with a projected size of $\sim 10 \times 36 \mathrm{kpc}^{2}$. The local HI peak in this feature is $\sim 3 \times 10^{19} \mathrm{~cm}^{-2}$, well below the star formation threshold (Kennicutt 1998; Bigiel et al. 2008). The HI flux 

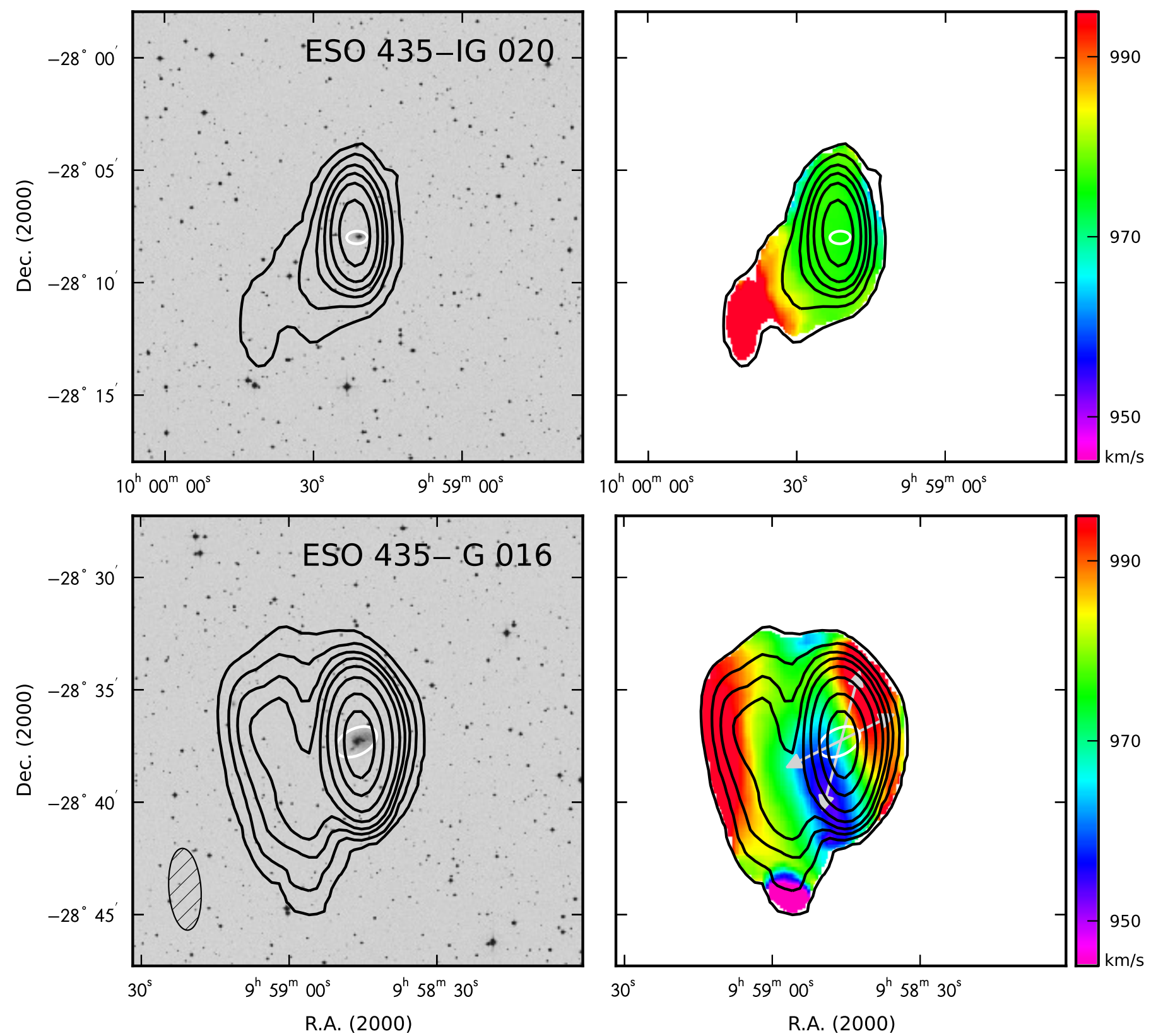

Fig. 4. HI intensity map overlaid on the DSS blue image (left) and velocity field (right). The HI contour levels are $(0.6,1.8,3,4.2,6,9.6,16.8$, $25.8) \times 10^{19} \mathrm{~cm}^{-2}$. The synthesized beam of our ATCA observation is shown on the bottom left corner and the velocity scale is shown by a color bar on the top right panel. We note that the velocity fields were generated by taking the first moments of the intensity-weighted channels. As the result, the maximum velocity of the northwest end in ESO 435- G 016 appears to be lower $\left(1000 \mathrm{~km} \mathrm{~s}^{-1}\right)$ than the highest velocity of the channel with emission $\left(\sim 1050 \mathrm{~km} \mathrm{~s}^{-1}\right)$. Two gray lines are presented to indicate the optically defined position angle (which agrees with the position angle of the inner HI disk) and the position angle of the outer HI disk. Further details are discussed in Sect. 5.3.

outside the symmetric gas disk is $\approx 23 \%$ of the total flux measured from this galaxy.

The total HI mass of individual systems is calculated using the following expression,

$$
M_{\mathrm{HI}}\left[M_{\odot}\right]=2.356 \times 10^{5} S_{\mathrm{HI}} d^{2},
$$

where $S_{\mathrm{HI}}$ is the integrated HI flux in $\mathrm{Jy} \mathrm{km} \mathrm{s}^{-1}$ and $d$ is the distance to the galaxy in Mpc. We adopt a distance of 9.0 Mpc and 9.1 Mpc for ESO 435-IG 020 and ESO 435-G 016, respectively (Kennicutt et al. 2008). The total HI masses measured from the ATCA data are $7.6 \times 10^{7} M_{\odot}$ and $3.1 \times 10^{8} M_{\odot}$, yielding $M_{\mathrm{HI}} / L_{B}$ of 0.38 and 0.52 for ESO 435-IG 020 and ESO 435-G 016. These are comparable to $M_{\mathrm{HI}} / L_{B}$ found in the BCD population
(Huchtmeier et al. 2007), but larger than normal spirals by a few factors (e.g., $M_{\mathrm{HI}} / L_{B} \approx 0.21$ for the entire sample of the HI Nearby Galaxy Survey (THINGS), and smaller when dwarf galaxies are excluded; Walter et al. 2008).

The gas peculiarities of the pair are also seen in their kinematics. At this velocity resolution, the HI disk of ESO 435-IG 020 barely shows rotation across the morphologically symmetric part. Along the gas tail however, the velocity changes quite steeply, exceeding the velocity range found in the main HI disk as shown in Fig. 4. The tail is largely responsible for the linewidth of this galaxy.

Meanwhile, ESO 435-G 016 shows clear rotation across the stellar disk, and out to a few optical radii. Within the stellar disk, 
Table 2. Measured HI properties of the targets.

\begin{tabular}{lcccccccc}
\hline \hline Galaxy & $\begin{array}{c}\Delta_{\mathrm{RA}} \\
-\operatorname{arcsec}-\end{array}$ & $\begin{array}{c}\Delta_{\text {Dec }} \\
\text { Jy km s }\end{array}$ & $\begin{array}{c}S_{\mathrm{HI}} \\
10^{8} M_{\odot}\end{array}$ & $\begin{array}{c}M_{\mathrm{HI}} \\
-\mathrm{km} \mathrm{s}^{-1}-\end{array}$ & $\begin{array}{c}V_{\mathrm{HI}} \\
M_{\odot} / L_{\odot}\end{array}$ \\
\hline ESO 435-IG 020 & 7 & 16 & $5.71 \pm 0.19$ & 1.11 & 100.1 & 63.4 & $973.0(974.0)$ & 0.38 \\
ESO 435-G 016 & 5 & 8 & $15.97 \pm 0.10$ & 3.12 & 133.9 & 93.7 & $973.2(974.9)$ & 0.52 \\
\hline
\end{tabular}

Notes. The 2nd and 3rd columns $\left(\Delta_{\mathrm{RA}}\right.$ and $\left.\Delta_{\mathrm{Dec}}\right)$ indicate the offset between the optical center and the peak of the HI emission. For HI mass, the distances in Table 1 have been applied. The HI velocity defined within the symmetric part of the gas disk is given within the parenthesis.

the kinematical major axis measured using the tilted ring model is 112 degrees in PA, being more or less consistent with the optically defined major axis (117 degrees) but with the HI peak offset from the optical center (see Table 2). Beyond the optical disk however, the velocity structure becomes warped, with the outer disk PA deviating from the inner disk by $\sim 50$ degrees (PA $~ 160$ degrees). Across the eastern extension, the velocity changes by $>\sim 50 \mathrm{~km} \mathrm{~s}^{-1}$, with the same velocity gradient as the receding side as well as the tail of ESO 435-IG 020, as seen in Fig. 4. Unlike the tail of the other galaxy however, this extension does not exceed the velocity coverage of the main HI disk. If the gas is on its way out, the stripping may have started recently and the gas has not been accelerated more than the escape velocity of the galaxy. On the other hand, if the gas is of external origin, the accretion might have been going for a while to require a similar velocity range to the main gas disk. Alternatively, the gas which had been stripped from the past interaction might be currently falling back.

The HI linewidths are measured at the velocities where the HI flux drops to $20 \%$ and $50 \%$ of the peak in HI profile for each galaxy (Fig. 2). Neither has a linewidth measured at $50 \%$ of the peak larger than $150 \mathrm{~km} \mathrm{~s}^{-1}$, implying that these galaxies are unlikely to have gone through a major merging recently (Sung et al. 2002). However, this still does not rule out the possibility of minor merging or tidal perturbation, not only by other neighbors but also by each other.

HI measurements including HI mass, linewidth, and the velocity can be found in Table 2 . The HI velocity has been measured both across the entire HI disk, and within the main disk of symmetric morphology.

\section{Discussion}

\subsection{Intergalactic gas between the pair}

In spite of a number of morphological and kinematical peculiarities in HI associated with both galaxies, we do not find any HI gas emission between the pair in the ATCA data down to $\approx 10^{19} \mathrm{~cm}^{-2}$ at the $3 \sigma$ level (assuming the linewidth of $50 \mathrm{~km} \mathrm{~s}^{-1}$ ). Then the question is whether the HI gas bridge revealed by the HIPASS survey is real, or is it simply the HIPASS resolution which makes them appear to be connected. In fact, the total ATCA flux is only $\sim 61 \%$ of the HIPASS flux, and it is possible that large-scale structures with low density between the pair have been resolved out in the ATCA observations. If the HI gas between the pair found in the HIPASS data is indeed real rather than the results of an overlap between two unresolved gas blobs, we might be able to extract the intergalactic HI gas from the HIPASS data.

In order to verify the presence of HI gas between the pair, and also to assess its flux, we have compared the HIPASS and the ATCA image in the same resolution as the HIPASS data, using the following procedure. First, the ATCA image was

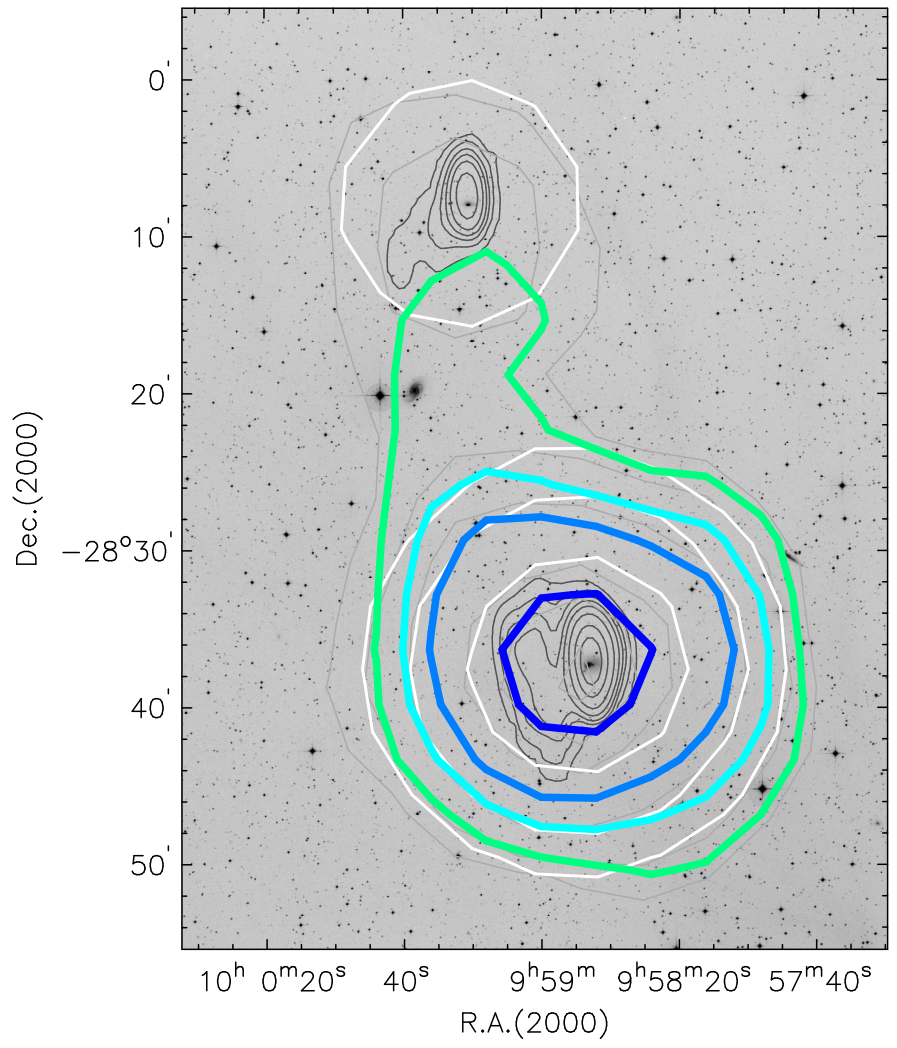

Fig. 5. HIPASS and ATCA HI maps are represented by contours overlaid on the DSS red optical image with the residual emission between them. The contours of the HIPASS and the high-resolution ATCA images are shown in light- and dark-gray, respectively. The levels are the same as in Figs. 1 and 4. The smoothed and regridded ATCA image is shown in white contours at the same levels as the HIPASS data. The residual emissions of $(1,2,3,5) \times \sigma$ are shown in green, cyan, lightblue, and blue, respectively, which are comparable to $(2.5,5,7.5,12) \times$ $\sigma$ of the HIPASS data.

convolved to the same resolution as the HIPASS image, from $218 \times 85 \operatorname{arcsec}^{2}$ to $930 \times 930 \operatorname{arcsec}^{2}$. Then the convolved ATCA image was re-gridded to the same cell size as the HIPASS image from 20 arcsec to 240 arcsec.

The result is presented in Fig. 5. The HI emission centered on ESO 435-IG 020 in the convolved ATCA data is offset by $\approx 2.4$ arcmin from the nearest local peak around this galaxy in the HIPASS data. Considering that the positional accuracy of the HIPASS survey is $\sim 1$ arcmin or so (Zwaan et al. 2004), this offset is likely to be real. However, as previously mentioned, the total flux measured from the ATCA data is consistent with the flux from the single-dish measurement, and hence the residual around this galaxy is below $1 \sigma$ of the residual image.

Instead, most of the missing flux is found around ESO 435-G 016, as expected from the comparison of previous 
Table 3. Multi-wavelength observations used in SED fitting.

\begin{tabular}{|c|c|c|}
\hline Band & $\begin{array}{c}\text { ESO 435-IG 020 } \\
\text { Flux density (Jy) or luminosity }\end{array}$ & Reference \\
\hline FUV (1515 ̊) & $1.91( \pm 0.19) \times 10^{-3}$ & 1 \\
\hline NUV (2273 ̊) & $3.22( \pm 0.32) \times 10^{-3}$ & 1 \\
\hline $\mathrm{H} \alpha$ (luminosity) & $1.25 \times 10^{40}\left(\mathrm{ergs} \mathrm{s}^{-1}\right)$ & 2 \\
\hline$B$ & $6.99( \pm 0.69) \times 10^{-3}$ & 3 \\
\hline$R$ & $1.13( \pm 0.11) \times 10^{-2}$ & 3 \\
\hline$J$ & $1.15( \pm 0.05) \times 10^{-2}$ & 4 \\
\hline$H$ & $1.07( \pm 0.08) \times 10^{-2}$ & 4 \\
\hline$K$ & $6.89( \pm 0.91) \times 10^{-3}$ & 4 \\
\hline $3.6 \mu \mathrm{m}$ & $5.72( \pm 0.17) \times 10^{-3}$ & 5 \\
\hline $4.5 \mu \mathrm{m}$ & $3.91( \pm 0.12) \times 10^{-3}$ & 5 \\
\hline $5.6 \mu \mathrm{m}$ & $2.32( \pm 0.11) \times 10^{-3}$ & 5 \\
\hline $8.0 \mu \mathrm{m}$ & $5.68( \pm 0.19) \times 10^{-3}$ & 5 \\
\hline $24 \mu \mathrm{m}$ & $7.59( \pm 0.16) \times 10^{-2}$ & 5 \\
\hline $70 \mu \mathrm{m}$ & $9.35( \pm 0.47) \times 10^{-1}$ & 5 \\
\hline \multirow[t]{2}{*}{$160 \mu \mathrm{m}$} & $5.31( \pm 0.76) \times 10^{-1}$ & 5 \\
\hline & ESO 435- G 016 & \\
\hline FUV (1515 Å) & $1.08( \pm 0.11) \times 10^{-3}$ & 1 \\
\hline NUV (2273 A) & $2.15( \pm 0.22) \times 10^{-3}$ & 1 \\
\hline$B$ & $1.81( \pm 0.16) \times 10^{-2}$ & 6 \\
\hline$R$ & $3.88( \pm 0.34) \times 10^{-2}$ & 6 \\
\hline$J$ & $6.11( \pm 0.27) \times 10^{-2}$ & 4 \\
\hline$H$ & $7.09( \pm 0.35) \times 10^{-2}$ & 4 \\
\hline$K$ & $5.70( \pm 0.39) \times 10^{-2}$ & 4 \\
\hline $65 \mu \mathrm{m}$ & $3.79( \pm 1.33)$ & 7 \\
\hline $90 \mu \mathrm{m}$ & $2.13( \pm 0.64)$ & 7 \\
\hline $140 \mu \mathrm{m}$ & $4.55( \pm 2.73)$ & 7 \\
\hline
\end{tabular}

References. Key to Col. 3: 1 - remeasured by this work; 2 Kennicutt et al. (2008); 3 - Gil de Paz et al. (2003); 4 - Skrutskie et al. (2003); 5 - Engelbracht et al. (2008); 6 - Lauberts \& Valentijn (1989); 7 - Pollo et al. (2010).

single-dish measurements and our ATCA fluxes of individual galaxies (see Sect. 4.1). The total residual flux that is likely to be associated with this galaxy (i.e., Dec $\lesssim-28^{\circ} 24^{\prime}$ ) accounts for $\sim 89 \%$ of the missing flux. Intriguingly, the remaining $\sim 10 \%$ is found in the intergalactic space between the lowest contours around the two galaxies $\left(-28^{\circ} 24^{\prime} \lesssim\right.$ Dec $\left.\lesssim-28^{\circ} 15^{\prime}\right)$. Although the net residual emission around ESO 435-IG 020 is insignificant, the offset of the HIPASS peak to the south from that of the ATCA data also supports the possibility that the intergalactic HI gas between the two galaxies is likely to be real.

\subsection{Star formation histories}

We study the star formation properties of this pair of BCDs in this section using archival optical, ultraviolet, and infrared observations. The far-ultraviolet (FUV) and near-ultraviolet (NUV) imaging data of our targets are obtained from the Galaxy Evolution Explorer (GALEX) UV satellite telescope's archive. Fortunately, there exists deep GALEX observations for this pair of galaxies. The integrated FUV-NUV colors (corrected for Galactic extinction) for ESO 435-G 016 and ESO 435-IG 020 are 0.65 and 0.43 in $\mathrm{AB}$ magnitudes, respectively. Mid- and far-infrared measurements are obtained using archival observations from the Spitzer and Akari space telescopes. The multiwavelength flux density measurements used in this section can be found in Table 3. Figure 6 overlays the FUV observations (orange contours) on the optical 3-color image derived from SuperCOSMOS (Hambly et al. 2001).
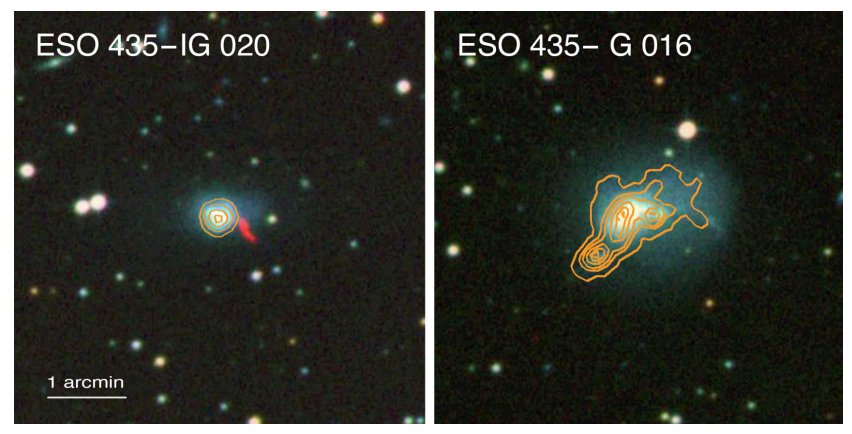

Fig. 6. FUV contours (orange) are overlaid on the SuperCOSMOS UKST $B j, R$, and $I 3$-color image. A scale bar of 1 arcmin is shown on the left bottom corner, which corresponds to $\approx 2.6 \mathrm{kpc}$ at a $9 \mathrm{Mpc}$ distance. The red tail in ESO 435-IG 020 is an imaging artifact in the I-band observations.

Due to the complex evolutionary history for this pair of BCDs, single stellar population models are insufficient for characterizing the star formation history of such galaxies. As such, we approximate and constrain the star formation history by fitting the observed spectral energy distributions (SED) from the far-ultraviolet to the far-infrared to model star formation histories using the Multi-wavelength Analysis of Galaxy Physical Properties software (MAGPHYS; da Cunha et al. 2008).

The observed multi-wavelength SEDs of ESO 435-IG 020 and ESO 435-G 016 are shown as red points in panels a of Fig. 7. For ESO 435-IG 020, the greatest difference between the observed SED and the model fit occurs in the $R$-band. As can be seen on the left of Fig. 7, the observed $R$-band flux density is greater than that from the model. We attribute this discrepancy in the $R$-band to the strong $\mathrm{H} \alpha$ emission that has been observed in this galaxy. Previous studies (e.g., Kennicutt et al. 2008) find the $\mathrm{H} \alpha$ luminosity to be $1.2 \times 10^{40} \mathrm{ergs} \mathrm{s}^{-1}$. According to the best fitting MAGPHYS model, ESO 435-IG 020 is consistent with a very low stellar mass dwarf galaxy that is currently forming stars with high efficiency as indicated by the SSFR value of $4.5 \times 10^{-8} \mathrm{yr}^{-1}$. For comparison, the average sSFR value for nearby star-forming galaxies is $3.2 \times 10^{-9} \mathrm{yr}^{-1}$ (Wong et al. 2016). Therefore, ESO 435-IG 020 is more efficient at forming stars by nearly an order of magnitude relative to other star-forming nearby galaxies. The model finds that it has been approximately 1.7 Gyr since the last burst of star formation in this galaxy. Similarly, the HI-normalized star formation efficiency $\left(S F E_{\mathrm{HI}}=S F R / M_{\mathrm{HI}}\right)$ for ESO 435-IG 020 is approximately four times greater than the average found in nearby starforming galaxies (Wong et al. 2016).

Conversely, the best fit models find that ESO 435-G 016 is likely to be 50 times more massive in stellar mass. ESO 435-G 016 has an average star formation rate over the last $10 \mathrm{Myr}$ and $2 \mathrm{Gyr}$ that are factors of 2.3 and 4.2 less than that for ESO 435-IG 020, respectively. The best fit model suggests that it has been $5.3 \mathrm{Gyr}$ since the last burst of star formation ended for ESO 435-G 016. The best fit model finds a median SSFR that is a factor of 2.7 less than the average nearby star-forming galaxy of similar stellar mass. The $S F E_{\mathrm{HI}}$ for ESO 435-G 016 is very similar to the average value found for nearby star-forming galaxies (Wong et al. 2016).

Further constraints on the star formation history on shorter timescales (a few Myr) are possible via the comparison of $\mathrm{H} \alpha$ to FUV observations. However, archival $\mathrm{H} \alpha$ observations are not available for ESO 435-G 016. As such, we can only directly confirm that there is a significant population of very young 

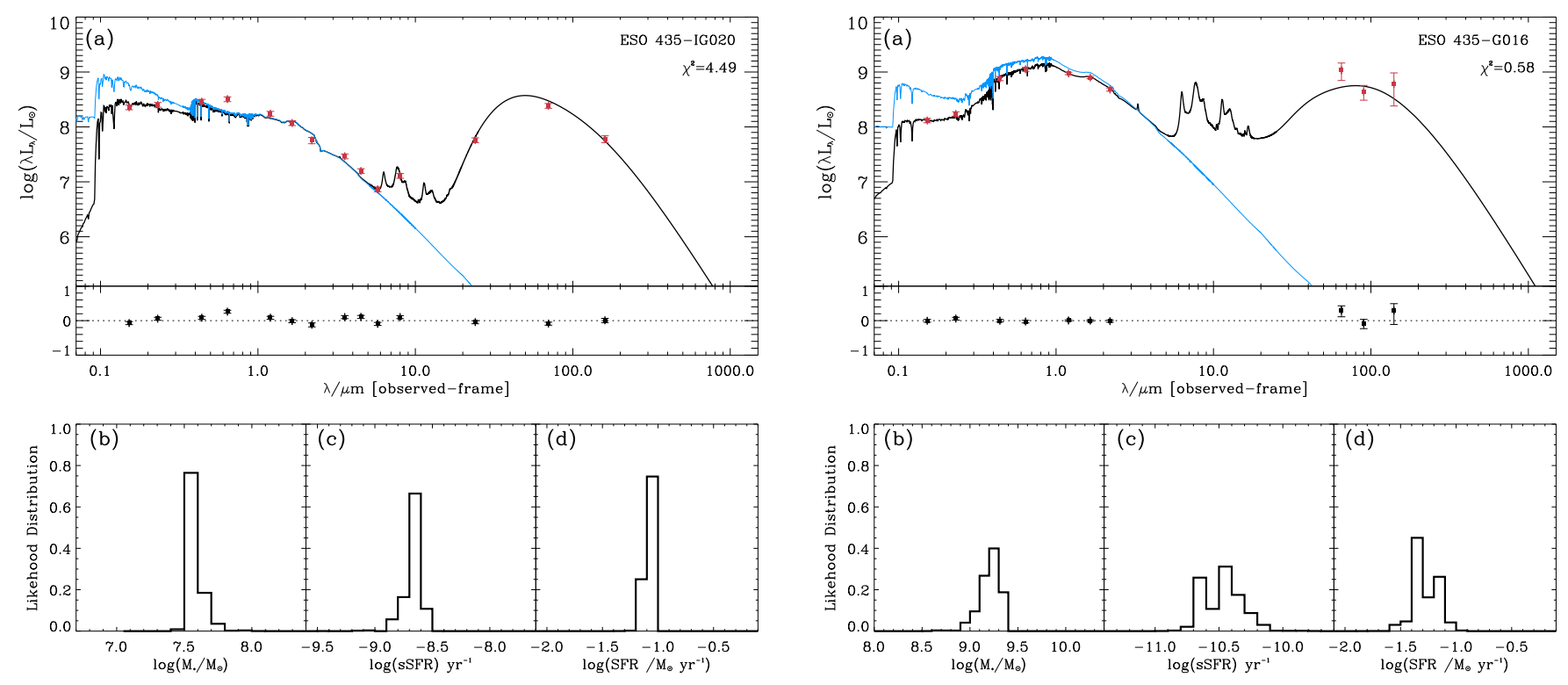

Fig. 7. Observed multi-wavelength SED of ESO 435-IG 020 and ESO 435- G 016 are shown as red points in panels $a$ on the left and on the right, respectively. The thick black solid lines represent the best fitting SED model estimated by MAGPHYS and the thin blue solid line represents the spectrum for the unattenuated stellar population. The goodness-of-fit is indicated by the $\chi^{2}$ value shown in panels a. For both galaxies, panels $b-d$ show the resulting likelihood distributions for the stellar mass, specific star formation history (sSFR), and the star formation rate (SFR) for each of the modeled SED.

stars $(<10$ Myr) in ESO 435-IG 020. This is consistent with the strong "bursty" star formation history that we found for ESO 435-IG 020 via SED modeling. Future $\mathrm{H} \alpha$ observations of ESO 435-G 016 will be useful for estimating its star formation history on shorter timescales. However, we can already tell from the SED modeling and SSFR that it is less bursty and less intense than that of ESO 435-IG 020. Nevertheless, what makes ESO 435-G 016 still interesting is its kinematical structures which indicate that this system is currently under the influence of the tidal force - potentially the origin of its recent active star formation, as described in the following section.

\subsection{Interaction history}

As argued in 5.1, the presence of intergalactic HI gas between the pair is somewhat likely. Also, as seen in the high-resolution ATCA data, both galaxies reveal a number of peculiarities in their HI distribution as well as in their optical morphology. All these strongly suggest that they have been tidally disturbed, potentially due to each other but also possibly by other neighbors.

In order to investigate the feasibility of tidal interaction with other neighboring galaxies, we probe the environment around the pair of $\sim 0.5 \mathrm{Mpc}$ radius within $\pm 300 \mathrm{~km} \mathrm{~s}^{-1}$, that is, a comparable size and velocity coverage of a typical galaxy group like our own. Within given ranges, there are 12 optically identified galaxies and several galaxy groups. Both ESO 435-IG 020 and ESO 435-G 016 are classified as the members of the NGC 3056 triplet, which is located in the outskirts of the NGC 3175 group (Makarov \& Karachentsev 2009, 2011). Among these, we find only two sizeable galaxies that could have tidally perturbed the pair, NGC 3056 and NGC 3113. In $I$-band they are brighter than ESO $435-\mathrm{G} 016$ by $\sim 0.9$ mag and $\sim 1.3 \mathrm{mag}$, respectivey (Doyle et al. 2005; Springob et al. 2007).

The distances from NGC 3056 and NGC 3113 to the center of the pair are $\sim 154 \mathrm{kpc}$ and $196 \mathrm{kpc}$, assuming the same distance to the pair, that is, $9 \mathrm{Mpc}$. Adopting $300 \mathrm{~km} \mathrm{~s}^{-1}$, a typical galaxy speed in groups, the crossing time between the pair and these two relatively massive galaxies is $480-640 \mathrm{Myr}$ or so. This is shorter than the timescale for the last burst of star formation which is likely to be responsible for the dominant young stellar population in both galaxies ( $>1 \mathrm{Gyr}$ ). This implies that any of these neighbors is potentially responsible for the last burst of star formation, if it was caused by tidal interaction.

However, for a crossing time between these neighbors and our BCD galaxies to be comparable to the age of the youngest stellar population of ESO 435-IG 020 that is traced by $\mathrm{H} \alpha$, they should be moving with a relative velocity of an order of $\sim 1000 \mathrm{~km} \mathrm{~s}^{-1}$, which is unlikely in this environment. Therefore any of the close neighbors, including the two most massive galaxies, are unlikely to be the most recent active star formation in our BCD galaxies.

Instead, the interaction between the pair seems to be a more feasible scenario. Assuming the same velocity as their neighbors, the crossing time between these two BCD galaxies is $\sim 260 \mathrm{Myr}$, still too large to explain their recent active star formation. If the past encounter between the BCD pair was a flyby however, its impact might have been just strong enough to pull out the materials from the outer disk, mostly gas, without triggering strong starbursts in the inner region of the galaxies. Since the close approach, some of the tidally stripped gas may have been recaptured (e.g., Duc \& Renaud 2013). Then stars can be actively formed by the reaccreted gas directly funneling into the galaxy than by generating shocks into the halo gas of the host galaxy, which is more likely for low mass systems (e.g., Benson \& Bower 2010).

At the same time, this accretion-induced star formation activity can be aided by the torque and shear on the gas. Indeed, the kinematical structure of ESO 435-G 016's HI disk is quite intriguing. While the position angle (PA) of the inner $\mathrm{HI}$ is more or less consistent with the optically defined position angle (PA), the angle connecting the local velocity maxima in the receding and approaching side appears to be tilted counterclockwise from the inner PA. In order to measure the offset more quantitatively, 
we modeled a cube of a rotating disk using GALMOD, a package implemented in GIPSY2.

As a result, the modeled kinematic structure based on the optical morphology in the inner region of the main HI disk agrees well with the observed velocity field, as indicated by an ellipse and a gray line along the optical major axis in Fig. 4. In the outer disk however, a second rotating component is present along the axis tilted counterclockwise from the inner kinematic axis. The position angle of the second kinematic component measured from the model, $\mathrm{PA} \approx 160^{\circ}$, well matches the axis connecting the local velocity maxima in the outer HI disk, as shown by the second gray line in Fig. 4.

The offset between the inner and the outer kinematic axes might have been caused by the interaction with ESO 435-IG 020, which could induce the shear. The speculation that the outer gas disk is likely to have been tidally dragged counterclockwise is also supported by a recent ALMA (Atacama Large Millimeter Array) observation by Ueda et al. (2014, AM 0956-282 in their sample). ESO 435-G 016 is detected in ${ }^{12} \mathrm{CO}(J=1-0)$ in the inner $\sim 1.6 \mathrm{kpc}$ radius. Intriguingly, the $\mathrm{CO}$ disk also has two position angles. The outer part of the $\mathrm{CO}$ disk, which is composed of the northwest extent and the southeast cloud, is more tilted to the counterclockwise in comparison to the inner $\mathrm{CO}$ disk. Especially cool gas components are expected to be influenced more effectively by torque due to their low velocity dispersion and collisional dynamics (e.g., Bournaud 2011). Hence the fact that the kinematical axis of the outer $\mathrm{CO}$ disk is rotated counterclockwise like the outer HI disk, is strongly suggestive of the presence of such force in this galaxy. Ueda et al. (2014) also propose that the southeast CO cloud is likely to have been ejected from the main molecular gas disk due to the interaction.

In Fig. 8 we illustrate a possible interaction scenario of the pair based on all the observational evidence and the environment of the pair. In this scenario, the pair have gone through a close encounter a while ago ( $\sim$ a few 100 Myr ago assuming typical group environment), which tidally stripped gas from the outer region. The intergalactic HI hinted at by the HIPASS data must be part of the stripped gas from the first encounter.

It is difficult to judge whether the orbit is closed or not, but if ESO 435-IG 020's tail was formed via fly-by interaction between the pair, its direction suggests that the two galaxies are in situ moving apart based on some simulations where tidal materials are found to form in retrograde after the first encounter in fly-by interactions (e.g., Pawlowski et al. 2011). Likewise it is hard to tell whether the tail of ESO 435-IG 020 and ESO 435-G 016's extension are being stripped or reaccreted. However, accretion is more feasible if the two galaxies are currently moving away, and their recent bursts of star formation can be also more naturally explained.

Even if the two galaxies are moving apart as suggested by ESO 435-IG 020's tail, the velocity shear across the main HI disk implies that the two galaxies are still under the influence of each other. The $\mathrm{CO}$ disk also shows at least two kinematic axes, with the outer position angle rotated in the same direction as the outer HI kinematic axis, supporting the presence of torque in the gas disk. Although the shear is less visible in ESO 435-IG 020 due to its compact structure and its nature that is less rotationally supported compared to ESO 435-G 016, it must be also experiencing the torque as the interaction counterpart of

\footnotetext{
2 Groningen Image Processing System, https://www.astro.rug. nl/ gipsy/
}

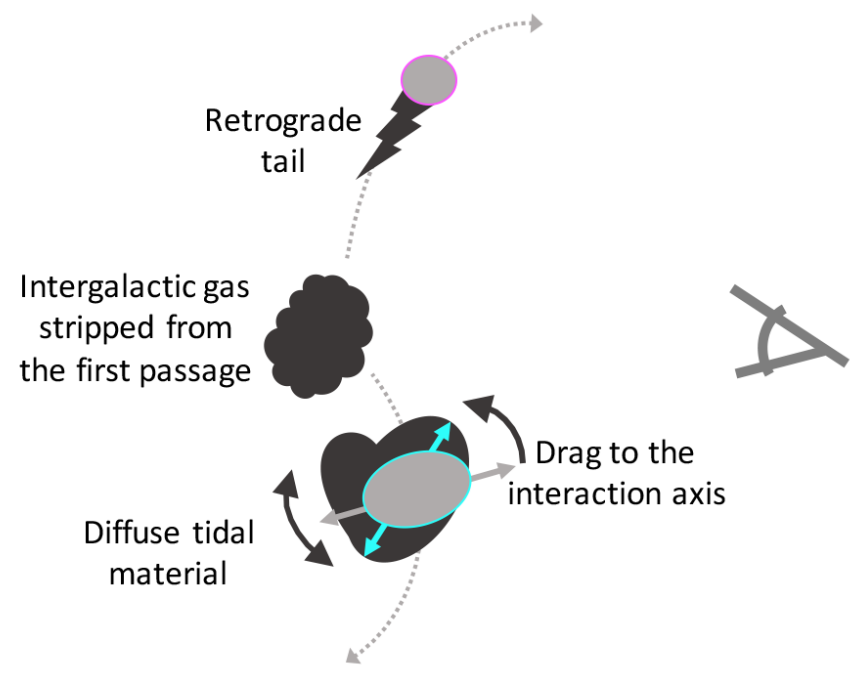

Fig. 8. A possible interaction scenario of the pair. A few $100 \mathrm{Myr}$ ago, the two galaxies may have gone through a close encounter, tidally pulling out the gas from their outer disks. The intergalactic HI hinted by the HIPASS data must be part of the tidally stripped gas. Based on the direction of the gas tail of ESO 435-IG 020 and the star formation/interaction timescale between the pair, two galaxies are inferred to be moving away from each other rather than being on the way to the second encounter. However, the main kinematic axis might still be dragged toward the interaction counterpart, generating torque on the disk (more effectively on the gas component), which can be clearly seen only in ESO 435-G 016 where the structure is better resolved than ESO 435-IG 020, at the resolution probed in this work. The recent bursts of star formation are likely to have been triggered by the shear on the gas disk and/or the re-accretion of the stripped gas.

ESO 435-G 016. This shear could be responsible for recent enhanced star formation activity in both galaxies.

Lastly, it is worth mentioning that we cannot completely rule out the merging between gas-rich dwarf galaxies as the origin of the recently enhanced star formation in these BCDs. A nucleated core and diffuse structures do well agree with BCDs originating from the merging of gas-rich galaxies simulated by Bekki (2008). In Bekki (2008)'s simulation, however, only the old stars with an age $>1$ Gyr and with gas form diffuse low-surface brightness structures, and new stars mostly form in the central region after merging. However, this is not the case of our pair as shown by the FUV image in Fig. 6, especially of ESO 435-G 016, where the internal structures can be better seen.

\section{Summary and conclusion}

We analyzed the HI imaging data of a BCD pair, ESO 435-IG 020 and ESO 435-G 016, obtained using the ATCA. The goal was to find the evidence for fly-by interaction between the pair as suggested by a large HI envelope covering both that is found by the HIPASS, and to investigate the role of tidal interaction in triggering recent active star formation in the pair.

From high resolution HI imaging data, we find clear indications that both galaxies have been tidally disturbed. However, intergalactic HI gas is not detected with the sensitivity limit of the ATCA observaions, which has recovered only $61 \%$ of the HIPASS HI flux. Considering typical galaxy speeds in groups, there are only two candidates which are massive enough to gravitationally perturb the pair in their neighborhood. The crossing time between the BCD pair and the neighbors within a radius 
of a comparable size to typical galaxy groups is short enough to explain the mean age of the dominant young stellar population ( $>1 \mathrm{Gyr}$ ), while it is still too large to be responsible for the most recent active star formation event in our BCDs.

Intriguingly, the residual emission between the HIPASS and the ATCA HI data is mainly found between the two galaxies. In addition, we found kinematical evidence for the shear on the gas disk. The second kinematic axis underlying the main HI disk of ESO 435-G 016 indicates that the gas component is under the influence of torque, which is also supported by the kinematics of its ${ }^{12} \mathrm{CO}(J=1-0)$ gas disk.

Based on our analysis, we conclude that the interaction between the two galaxies is responsible for their HI and optical peculiarities as well as the recent star formation activities. During the most recent encounter, the pair could have lost HI gas from the outer disk. Enhancements in recent star formation on timescales of $\sim 10$ Myr might have been triggered by the reaccretion of the gas that had been stripped during the past encounter and/or the shear on the disk caused by each other.

In this study of a BCD pair, we have provided observational evidence that star formation can be enhanced by fly-by interaction between galaxies without merging. If this well-separated pair of BCDs are representative of BCDs, we propose that fly-by interactions could be one of the most important origins of BCDs. Our suggestion can explain the observation that a high fraction of BCDs are not in the merging process ( $60 \%$, e.g., Sung et al. 2002).

Acknowledgements. We are grateful to the anonymous referee for his/her valuable comments and useful suggestions. Support for this work was provided by the National Research Foundation of Korea to the Center for Galaxy Evolution Research (No. 2010-0027910) and Science Fellowship of POSCO TJ Park Foundation. This work has been also supported by NRF grant No. 2015R1D1A1A01060516. Parts of this research were conducted by the Australian Research Council Centre of Excellence for All-sky Astrophysics (CAASTRO), through project number CE110001020. We are grateful to Bärbel Koribalski for her useful suggestions and comments. The Australia Telescope Compact Array is part of the Australia Telescope National Facility which is funded by the Australian Government for operation as a National Facility managed by CSIRO. This work has made use of the NASA/IPAC Extragalactic Database (NED) which is operated by the Jet Propulsion Laboratory, California Institute of Technology, under contract with the National Aeronautics and Space Administration. We thank Elisabete da Cunha and Simon Driver for their helpful guidance with MAGPHYS

\section{References}

Aloisi, A., Tosi, M., \& Greggio, L. 1999, AJ, 118, 302

Bekki, K. 2008, MNRAS, 388, L10

Benson, A. J., \& Bower, R. 2010, e-print ArXiv [arXiv: 1004 . 1162]

Bigiel, F., Leroy, A., Walter, F., et al. 2008, AJ, 136, 2846

Bournaud, F. 2011, EAS Publ. Ser., 51, 107

Briggs, D. S. 1995, Ph.D. Thesis, New Mexico Institute of Mining and Technology, Socorro, NM

Cannon, J. M., Skillman, D., Kunth, D., et al. 2004, ApJ, 608, 768

Chengalur, J. N., Pustilnik, S. A., Martin, J.-M., \& Kniazev, A. Y. 2006, MNRAS, 371, 1849

Cloet-Osselaer, A., De Rijcke, S., Vandenbroucke, B., et al. 2014, MNRAS, 442, 2909

da Cunha, E., Charlot, S., \& Elbaz, D. 2008, MNRAS, 388, 1595

de Vaucouleurs, G., de Vaucouleurs, A., Corwin, H. G., et al. 1991, Third Reference Catalogue of Bright Galaxies (New York: Springer-Verlag) (RC3)
Di Matteo, P., Combes, F., Melchior, A.-L., \& Semelin, B. 2007, A\&A, 468, 61 Doublier, V., Caulet, A., \& Comte, G. 1999, A\&AS, 138, 213

Doyle, M. T., Drinkwater, M. J., Rohde, D. J., et al. 2005, MNRAS, 361, 34 Duc P.-A., \& Renaud F. 2013, in Lecture Notes in Physics, 861, eds. J. Souchay, S. Mathis, T. Tokieda (Berlin: Springer Verlag), 327

Ekta, B., Chengalur, J. N., \& Pustilnik, S. A. 2006, MNRAS, 372, 853 Engelbracht, C. W., Rieke, G. H., Gordon, K. D., et al. 2008, ApJ, 678, 804 Fisher, D. B., \& Drory, N. 2011, ApJ, 733, L47

Gil de Paz, A., Madore, B. F., \& Pevunova, O. 2003, ApJS, 147, 29

Hambly, N. C., MacGillivray, H. T., Read, M. A., et al. 2001, MNRAS, 326, 1279

Holmeberg, E. B., Lauberts, A., Schuster, H.-E., \& West R. M. 1974, A\&AS, 18, 463

Hopkins, A. M., Schulte-Ladbeck, R. E., \& Drozdovsky, I. O. 2002, AJ, 124, 862 Huchtmeier, W. K., Petrosian, A., Gopal-Krishna, \& Kunth, G. 2007, A\&A, 462, 919

Icke, V. 1985, A\&A, 114, 115

Kehrig, C., Telles E., \& Cuisinier, F. 2004, AJ, 128, 1141

Kennicutt, R. C. 1998, ApJ, 498, 541

Kennicutt, R. C., Lee, J. C., Funes, J. G., J. S., Sakai, S., \& Akiyama, S. 2008, ApJS, 178, 247

Koribalski, B. S., Staveley-Smith, L., Kilborn, V. A., et al. 2004, AJ, 128, 16

Kunth, D., \& Östlin, G. 2000, A\&ARv, 10, 1

Lauberts, A., \& Valentijn, E. A. 1989, The surface photometry catalogue of the ESO-Uppsala galaxies (Garching: European Southern Observatory)

Lelli, F., Verheijen, M., Fraternali, F., \& Sancisi, R. 2012, A\&A, 537, A72

López-Sánchez, Á. R., \& Esteban, C. 2009, A\&A, 508, 615

López-Sánchez, Á. R., Koribalski, B. S., van Eymeren, J., et al. 2012, MNRAS, 419, 1051

Makarov, D., \& Karachentsev, I. D. 2009, Astrophys. Bull., 64, 24

Makarov, D., \& Karachentsev, I. D. 2011, MNRAS, 412, 2498

Mendez, D. I., \& Esteban, C. 2000, A\&A, 359, 493

Meyer, M. J., Zwaan, M. A., Webster, R. L., et al. 2004, MNRAS, 350, 1195

Nidever, D. L., Ashley, T., Slater, C. T., et al. 2013, ApJ, 779, L15

Östlin, G., Amram, P., Masegosa, J., Bergvall, N., \& Boulesteix, J. 1999, A\&AS, 137,419

Östlin, G., Amram, P., Bergvall, N., et al. 2001, A\&A, 374, 800

Papaderos, P., Loose, H.-H., Fricke, K. J., \& Thuan, T. X. 1996, A\&A, 314, 59

Pawlowski, M. S., Kroupa, P., \& de Boer, K. S. 2011, A\&A, 532, A118

Pollo, A., Rybka, P., \& Takeuchi, T. T. 2010, A\&A, 514, A3

Pustilnik, S. A., Kniazev, A. Y., Lipovetsky, V. A., \& Ugryumov, A. V. 2001, A\&A, 373, 24

Rothberg, B., \& Joseph, R. D. 2004, AJ, 128, 2098

Rich, R. M., Collins, M. L. M., Black, C. M., et al. 2012, Nature, 482, 192

Salzer, J. J., MacAlpine, G. M., \& Boroson, T. A. 1989, ApJS, 70, 447

Sault, R. J., Teuben, P. J., \& Wright, M. C. H. 1995, in Astronomical Data Analysis Software and Systems IV. eds. R. Shaw, H. E. Payne, J. J. E. Hayes (San Francisco: ASP), ASP Conf. Ser., 77, 433

Searle, L., Sargent, W. L. W., \& Bagnuolo, W. G. 1973, ApJ, 179, 427

Skrutskie, M. F., Cutri, R. M., Stiening, R., et al. 2003, VizieR Online Data Catalog: VII/233

Springob, C. M., Masters, K. L., Haynes, M. P., Giovanelli, M. P., \& Marinoni, C. 2007 , ApJS, 172, 599

Sung, E.-C., Chun, M.-S., Freeman, K. C., \& Chaboyer, B. 2002, in The Dynamics, Structure \& History of Galaxies: A Workshop in Honour of Professor Ken Freeman, eds. G. S. Da Costa, E. M. Sadler, \& H. Jerjen, ASP Conf. Ser., 273, 341

Taylor, C. L. 1997, ApJ, 480, 524

Taylor, C. L., Brinks, E., Grashuis, R. M., \& Skillman, E. D. 1995, ApJS, 99, 427

Theureau, G., Coudreau, N., Hallet, N., et al. 2005, A\&A, 430, 373

Thuan, T. X. 1983, ApJ, 268, 667

Thuan, T. X., \& Martin, G. E. 1981, ApJ, 247, 823

Ueda, J., I., Daisuke, Y., Min S., et al. 2014, ApJS, 214, 1

van Zee, L., Skillman, E. D., \& Salzer, J. J. 1998, AJ, 116, 1186

Verbeke, R., De Rijcke, S., Koleva, M., et al. 2014, MNRAS, 442, 1830

Walter, F., Brinks, E., de Blok, W. J. G., et al. 2008, AJ, 136, 2563

Wong, O. I., Meurer, G. R., Zheng, Z., et al. 2016, MNRAS, 460, 1106

Zwaan, M. A., Meyer, M. J., Webster, R. L., et al. 2004, MNRAS, 350, 1210 